\title{
The long-range effect induced by untying hydrogen bonds for single cell test using SECM
}

Qing Zheng, ${ }^{\dagger}$ Yifan Yang, ${ }^{\dagger}$ Yuanyuan Yan, ${ }^{\dagger}$ Yu Yu, ${ }^{\ddagger}$ Yao Liu, ${ }^{\dagger}$ Wenyu Gao, ${ }^{\ddagger}$ Kejian Ding, ${ }^{*,+}$ and Huibo Shao ${ }^{*, \dagger}$

†Key Laboratory of Cluster Science (Ministry of Education) and Beijing Key Laboratory of Photoelectronic and Electrophotonic Conversion Materials, School of Chemistry, Beijing Institute of Technology, Beijing 100081, P.R. China

$\$$ College of Life sciences and Bioengineering, Beijing Jiaotong University, Beijing 100044, P. R. China

* Corresponding authors

E-mail addresses: hbs@bit.edu.cn (Huibo Shao), dkjian@bjtu.edu.cn (Kejian Ding).

\section{ABSTRACT:}

The enhanced positive feedback current of scanning electrochemical microscopy (SECM) is observed at a large separation between the tip and the substrate surface in the case of a 3-mercaptopropionic acid (3-MPA) self-assembled monolayer (SAM). The effect that induces the large separation (named the long-range effect) is confirmed by SECM and quantitatively studied by electrochemical quartz crystal microbalance (EQCM). A key finding is that the long-range effect results from untying hydrogen bonds beforehand formed between the hydroxyl group of tip redox species and the carboxyl group of 3-MPA SAM. It is found that the long-range effect is increased with the packing density of the 3-MPA SAM. On the basis of these findings, 
the long-range effect is applied to the nondestructive test of living cells using SECM.

\section{Introduction}

Scanning electrochemical microscopy (SECM) has evolved as a successful test technique for characterization and electrochemical kinetic study of surfaces $[1,2]$. Enhancing the feedback current of SECM, especially the positive feedback current, is one of the main directions of research over the past decade $[3,4]$. The in-situ surface interrogation mode of scanning electrochemical microscopy (SI-SECM) operates a transient positive feedback current for the characterization of adsorbed species on the substrate electrodes, providing opportunities for fundamental breakthroughs in electrochemistry $[5,6]$. This mode of SECM requires a current impulse to activate the adsorbed species on the substrate, and the tip-substrate separation (d) is less than one radius of the tip (a), d/a $<1$ [7]. This approach is however not ideal for surface investigations. Hence a novel approach that allows a larger tip-substrate separation and provides a sustainable positive feedback current, would have a wider significance on SECM. In these respects, it is necessary to create a new way to extend the diffusion distance of redox species and increase the concentration of redox species in front of the tip.

The self-assembled monolayer (SAM) bonded on all kinds of material surfaces, has become increasingly popular and better known over the past twenty years [8-11]. The bio-applications of SAM have been recognized and studied extensively [12-15]. This suggests enormous potential of the functional SAMs for clinical applications. The physical adsorption, covalent and covalent coordinate bond methods have been 
developed for molecules immobilization onto SAM [16, 17], such as covalent immobilization of proteins [18]. Among the intermolecular interactions developed for molecules immobilization on SAM, those based on weak interactions still need to explore [19].

Hence a new way is desirable to take advantage of the long-range interactions (e.g. hydrogen bonding) between the tip redox species and terminal groups of SAM on the substrate electrode. In consideration of the long-range interactions, ferrocenemethanol (described as $\mathrm{FcCH}_{2} \mathrm{OH}$ ) is selected as the tip redox species. This redox species benefits the formation of hydrogen bonding and electrostatic attraction because of the obvious distinction of electric charge between the reduced form $\left(\mathrm{FcCH}_{2} \mathrm{OH}\right)$ and the oxidized form $\left(\mathrm{Fc}^{+} \mathrm{CH}_{2} \mathrm{OH}\right)$. Besides, the hydroxyl group in $\mathrm{FcCH}_{2} \mathrm{OH}$ molecule provides the foundation for formation of hydrogen bonds [20]. It is common to graft molecules via intermolecular interactions with the aid of modified layers [21, 22]. The 3-mercaptopropanoic acid (described as 3-MPA) is one of the popular functional molecules to graft protein molecules, and possesses a carboxyl group in the terminal end [23].

If the hydrogen bonding interaction between $\mathrm{FcCH}_{2} \mathrm{OH}$ and 3-MPA is able to improve the concentration of redox species on the substrate surface and then release the redox species into the hemispherical diffusion profile of the tip by untying hydrogen bonds, the positive feedback current will be enhanced. That is to say, the tip could be positioned further to obtain a feedback current in the same intensity. This is beneficial for nondestructive test of living cells. Therefore, it 
becomes a useful strategy that initially adsorbs the tip redox species by hydrogen bonds and then releases them in the SECM scanning process by disturbing the hydrogen bonds.

In the neutral medium, like $\mathrm{pH} 7.0$, the ionization of the weak acid 3-MPA is feeble. The oxidation product of tip redox species, $\mathrm{Fc}^{+} \mathrm{CH}_{2} \mathrm{OH}$, facilitates the ionization of the carboxyl group by attracting the negative charged carboxylate ions. This leads to untying of hydrogen bonds between 3-MPA and $\mathrm{FcCH}_{2} \mathrm{OH}$. Consequently, the released $\mathrm{FcCH}_{2} \mathrm{OH}$ molecules will diffuse to the tip and enhance the feedback current of the tip. For these reasons, a new mode of SECM based on SAM modified substrate electrode is applied to our work. In the SECM experiment (Scheme 1), the substrate gold electrode is modified with SAM of 3-MPA which possesses a carboxyl group in the terminal end. $\mathrm{FcCH}_{2} \mathrm{OH}$ that equips with a hydroxyl group acts as the tip redox species. Before the test of the approach curve, a certain amount of tip redox species $\mathrm{FcCH}_{2} \mathrm{OH}$ are bonded on the 3-MPA SAM by hydrogen bonds. Interestingly, we find that the positive feedback current is enhanced, resulting from untying hydrogen bonds beforehand formed between the tip redox species and the 3-MPA SAM. The ionization of the carboxyl group is driven by the electrostatic attraction between $\mathrm{Fc}^{+} \mathrm{CH}_{2} \mathrm{OH}$ and the carboxylate ions. As a result, the released redox species diffuse into the range of the hemispherical profile in front of the tip. This brings to an interesting phenomenon that the tip feedback current of approach curve continuously increases in a very large tip-substrate separation. The effect that causes the feedback current increasing from 10 to 60 times radius of the micrometer tip is 
defined as the long-range effect. To the best of our knowledge, this is the first work to enhance the feedback current of SECM by using a SAM. Furthermore, the resulting long-range effect is applied to the single cell test using SECM which is beneficial for cell tests without affecting the natural metabolic pathway and cell morphology.

\section{Experimental}

\subsection{Chemicals and materials}

3-mercaptopropanoic acid (3-MPA), ferrocenemethanol $\left(\mathrm{FcCH}_{2} \mathrm{OH}\right)$, potassium ferricyanide, potassium ferrocyanide and potassium chloride were purchased from J\&K Scientific Co., Ltd., (Beijing, China). All chemicals were used with no further purification. Fetal bovine serum, Dulbecco's modified eagle's medium (DMEM) were purchased from Gibco Life Technologies (USA). Water (18.2 $\mathrm{M} \Omega \mathrm{cm}^{-1}$ ) used in the experiments was generated by a Milli-Q filter (Research UV, Hetai Instrument Co. Ltd., Shanghai, China). The gold disk electrode, $\mathrm{Ag} / \mathrm{AgCl}$ reference electrode and the platinum SECM tip with a radius of $5 \mu \mathrm{m}$ (Part number: $\mathrm{CHI116)}$ were purchased from $\mathrm{CH}$ instruments, Inc.

\subsection{Preparation of the substrate electrode with the 3-MPA SAM}

The polycrystalline gold disk electrodes were polished with alumina slurry to achieve a mirror-like surface and then cleaned by sonication. They were then washed with water and etched in $0.05 \mathrm{M} \mathrm{H}_{2} \mathrm{SO}_{4}$ solution by cycling the potential from 0 to 1.5 $\mathrm{V}$ (vs. $\mathrm{Ag} / \mathrm{AgCl}$, sat. $\mathrm{KCl}$ ) at a scanning rate of $50 \mathrm{mV} \mathrm{s}^{-1}$ and dried with pure nitrogen gas. 3-MPA SAM was prepared on clean surface of polycrystalline gold disk electrode. The procedure involved the immersion of the gold electrode into a $0.2 \mathrm{M}$ solution of 
3-MPA in ethanol at room temperature for 2 hours, followed by ultrasonic cleaning in water.

\subsection{Electrochemical measurements}

Electrochemical measurements were carried out with CHI920C scanning electrochemical microscopy. For cyclic voltammetry, a gold disk electrode was employed as the working electrode, a platinum wire as the counter electrode and an $\mathrm{Ag} / \mathrm{AgCl}$ electrode with saturated $\mathrm{KCl}$ solution as the reference electrode. For the SECM approach curve test, a micrometer Pt disk electrode of $10 \mu \mathrm{m}$ diameter was employed as the SECM tip and a 3-MPA SAM gold electrode as the substrate electrode. Electrochemical Impedance Spectroscopy measurements were performed in an electrochemical cell containing $5 \mathrm{mM} \mathrm{K}_{3}\left[\mathrm{Fe}(\mathrm{CN})_{6}\right]$ and $5 \mathrm{mM} \mathrm{K}_{4}\left[\mathrm{Fe}(\mathrm{CN})_{6}\right]$ in the frequency ranging from $100 \mathrm{kHz}$ to $1 \mathrm{~Hz}$ at $0.25 \mathrm{~V}$.

\subsection{EQCM analysis}

The electrochemical quartz crystal microbalance (EQCM) measurement of the self-assembling process of 3-MPA on gold substrate was carried out in QCM 922 (Princeton Applied Research) controlled by PARSTAT 4000 electrochemical work station. Prior to the experiment, the surface of QCM Au electrode was cleaned according to the manufacturer's protocol. After establishing a baseline for 40 minutes, 3-MPA (0.2 M solution in ethanol) was introduced into the QCM chamber. All the measurements were performed at room temperature. The increase in the mass of SAM was measured by Sauerbrey's equation [24]:

$$
\Delta \mathrm{m}=\frac{-A \sqrt{\rho \mu}}{2 F^{2}} \times \Delta \mathrm{F}
$$


Where $A$ is the surface area of the gold electrode $\left(A=0.2 \mathrm{~cm}^{2}\right), \rho$ is the density of quartz, $\mu$ is the shear modulus of quartz, $F$ is the inherent frequency $(9 \mathrm{MHz})$ of the quartz crystal and $\Delta \mathrm{F}$ is the frequency decrease of the quartz crystal.

\subsection{Cell culture and single cell test using SECM}

Cells were cultured in Dulbecco's modified-eagle medium (DMEM) supplemented with $10 \%$ fetal bovine serum (FBS) $\left(5 \% \mathrm{CO}_{2}, 37^{\circ} \mathrm{C}\right)$. After 48 hours, cells were trypsinized and collected. Prior to the SECM test, the cells were cultured in DMEM (lacking serum) containing $0.5 \mathrm{mM} \mathrm{FcCH}_{2} \mathrm{OH}$ for one hour as previously reported [25]. Biased at $0.4 \mathrm{~V}$ in $0.5 \mathrm{mM} \mathrm{FcCH}_{2} \mathrm{OH}$, the micrometer tip was positioned $\sim 30 \mu \mathrm{m}$ over the substrate electrode. The reported result for one-line cell scanning in this experiment was the mean value of measurements for ten individual cells, and the error bars represent the standard deviation $(n=10)$.

\section{Results and discussion}

Cyclic voltammetry (CV) and electrochemical impedance spectra (EIS) were carried out to prove the formation of SAM and describe the surface coverage of the SAM [26, 27]. Figure 1 shows the $\mathrm{CV}$ curves (a) and EIS (b) of $\left[\mathrm{Fe}(\mathrm{CN})_{6}\right]^{3-/ 4-}$ at the bare $\mathrm{Au}$ substrate electrode, 3-MPA SAM modified substrate electrode, respectively. The relevant electrochemical data are listed in Table 1. As can be seen, the redox species $\left[\mathrm{Fe}(\mathrm{CN})_{6}\right]^{3-/ 4-}$ at 3-MPA SAM substrate electrode presents one couple of reversible redox peaks which is similar with the redox species $\left[\mathrm{Fe}(\mathrm{CN})_{6}\right]^{3-/ 4-}$ at the bare $\mathrm{Au}$ substrate electrode. For 3-MPA SAM substrate electrode, both current of anodic peak 
$\left(I_{\mathrm{pa}}\right)$ and cathodic peak $\left(I_{\mathrm{pc}}\right)$ have a little decrease and the separation potential between anodic peak and cathodic peak $\left(\Delta E_{\mathrm{p}}\right)$ becomes bigger than the bare $\mathrm{Au}$ substrate electrode. In the experiment of EIS, the Randles equivalent circuit [28] was used to fit EIS data and determine the charge transfer resistance $\left(R_{c t}\right)$. The EIS curves show that the $R_{c t}$ value of 3-MPA SAM electrode $(282.1 \mathrm{ohm}$ ) is slightly higher than the bare electrode $(254.6 \mathrm{ohm})$. The results indicate the formation of the 3-MPA SAM. Further, the surface coverage $(\theta)$ of 3-MPA SAM was determined by comparing the area of the reduction peak of surface gold oxide $[29,30]$ in $0.1 \mathrm{M} \mathrm{H}_{2} \mathrm{SO}_{4}$ before and after modification which was calculated according to the equation (1).

$$
\theta=\frac{Q^{0}-Q}{Q^{0}} \times 100 \%
$$

Where $Q$ and $Q^{O}$ are the amount of electric charge exchanged during the electroadsorption of oxygen $\left(\mathrm{C} \mathrm{cm}^{-2}\right)$ of Au electrode with and without 3-MPA SAM respectively. The $\theta$ of the 3-MPA SAM modified $A u$ is calculated as $21.3 \%$ which indicates a low surface coverage of the SAM (Fig. 1c). This is reasonable because 3-MPA is short thiol molecule. More important, the lower surface coverage of the 3-MPA SAM makes the electrode suitable to serve as a substrate for SECM, because the surface defects of the 3-MPA SAM modified electrode allow electron transfer between the tip redox species and the substrate gold surface.

The micrometer tip electrode of $5 \mu \mathrm{m}$ radius was characterized by steady-state voltammetry. Figure 2(a) shows a normal steady-state voltammogram. The $\mathrm{S}$ shaped curve starts with a current close to zero and increases until it reaches a steady state that resulting from the limited diffusion of $\mathrm{FcCH}_{2} \mathrm{OH}$ to the tip. It proves that the tip 
electrode is satisfactory to SECM experiments. In order to validate the concentration increment of $\mathrm{FCCH}_{2} \mathrm{OH}$ molecules in front of the tip, as shown in Scheme 1, we designed a set of experiments considering whether there's hydrogen-bonding or not. The SECM approach curves approaching the bare gold electrode and 3-MPA SAM modified electrodes in $0.2 \mathrm{M} \mathrm{KCl}$ containing $0.5 \mathrm{mM} \mathrm{FcCH} \mathrm{m}_{2} \mathrm{OH}$ were performed respectively. As a control experiment, the conventional positive feedback approach curve (black line) and negative feedback approach curve (red line) of SECM were observed for the tips approaching a bare gold and an insulator surface respectively, seen in Figure 2(b). The positive feedback current starts with a steady current and sharply increases until the tip-substrate separation reaches 5 times radius of the tip. However, a positive feedback curve with a flat slope was observed for the 3-MPA SAM modified substrate, the tip-substrate separation was close to 60 times radius of the tip (blue line in Figure 2(b)) on the initial point of the flat slope. This experiment phenomenon is very different with the SECM approach curves obtained by the bare gold substrate and the alkyl (as terminal group) SAM modified substrates [31]. The positive feedback approach curve with a flat slope suggests that the additional tip redox mediator $\left(\mathrm{FcCH}_{2} \mathrm{OH}\right)$ has returned to the tip from outside the range of the hemispherical diffusion profile in front of the tip. The effect that causes the feedback current increasing from 10 to 60 times radius of the tip is defined as long-range effect. The result hints that the long-range effect is induced by untying hydrogen bonds beforehand formed between $\mathrm{FcCH}_{2} \mathrm{OH}$ and 3-MPA SAM. The experimental approach curve for the tip approaching an insulator surface is well fitted to the Cornut theory 
[32] (data not shown). However, the experimental approach curves for the tip approaching a 3-MPA SAM modified gold surface are difficult to fit because of the presence of the hydrogen bonding between 3-MPA SAM and tip redox species. So we take the positive feedback curve on gold as the "theoretical fitting" for positive feedback curve on 3-MPA SAM modified gold surface. The increment of feedback current for 3-MPA SAM modified gold surface is attributed to the long-range effect. In this way, the increment of feedback current induced by long-range effect can be quantified by the "theoretical fitting". Further, the approach curve towards 3-MPA SAM modified electrodes in $0.2 \mathrm{M} \mathrm{KCl}$ containing $2 \mathrm{mM} \mathrm{K}_{4}(\mathrm{FeCN})_{6}$ (as tip redox species) were performed (green line). The positive feedback current starts with a steady current until the tip-substrate separation is less than 5 times radius of the tip. This can be understood in consideration of tip redox species $\mathrm{K}_{4} \mathrm{Fe}(\mathrm{CN})_{6}$ which cannot form hydrogen bonds with 3-MPA. Therefore, there is no redox species $\mathrm{K}_{4} \mathrm{Fe}(\mathrm{CN})_{6}$ bonded on the 3-MPA SAM. As a result, the long-range effect was not observed in 0.2 $\mathrm{M} \mathrm{KCl}$ containing $2 \mathrm{mM} \mathrm{K}_{4} \mathrm{Fe}(\mathrm{CN})_{6}$.

The hydrogen bond, it is well known, could be hindered by the strong base or strong acid. So it is important to verify the proposed long-range effect under the strong basic or strong acidic condition. The SECM approach curves approaching 3-MPA SAM modified substrate under strong base or strong acid in $0.2 \mathrm{M} \mathrm{KCl}$ containing $0.5 \mathrm{mM} \mathrm{FcCH}{ }_{2} \mathrm{OH}$ were measured respectively. Shown in Figure 3(a), under strong basic condition ( $\mathrm{pH}$ 10.0), the positive feedback current starts with a steady current and increases until the tip-substrate separation reaches $\sim 5$ times of 
the tip radius. It suggests that the hydrogen bonds are disrupted and the ionization of the carboxyl group which is disadvantageous for hydrogen bonds formation, are also promoted. Consequently the SECM approach curve has returned to conventional shape and the long-range effect has disappeared. Under strong acidic condition ( $\mathrm{pH}$ 2.0), the positive feedback current starts with a steady current and increases until the tip-substrate separation reaches $\sim 60$ times of the tip radius, and the current increment is less than that under nearly neutral condition ( $\mathrm{pH} \mathrm{6.4).} \mathrm{In} \mathrm{this} \mathrm{case,}$ hydrogen bonds are also disrupted. However, the ionization of the carboxyl group is blocked by the hydrogen ions, which provides additional neutral 3-MPA molecules for hydrogen bonds formation. As a result, the long-range effect still exists but performs weaker than that under $\mathrm{pH}$ 6.4. These results further illustrate the importance of hydrogen bonds formation for the long-range effect.

Apparently, the feedback current is related to the diffusion behavior of tip redox species $\left(\mathrm{FcCH}_{2} \mathrm{OH}\right)$. The SECM approach curves recorded at different approach rates are shown in Figure 3(b), the approach curves obtained at the approach rates in the range of $0.5-50 \mu \mathrm{m} \mathrm{s}^{-1}$ are almost overlapped, and obviously show the long-range effect. This indicates that the diffusion behavior of tip redox species would not be affected by the motion of the tip electrode. So the approach rate of approach curve experiments in this work is set at $10 \mu \mathrm{m} \mathrm{s}^{-1}$ which is commonly used in SECM approaching test for a $10 \mu \mathrm{m}$ diameter tip [33].

Since the results show the diffusion behavior of tip redox species would not be affected by the motion of the tip electrode, the concentration gradients of $\mathrm{FcCH}_{2} \mathrm{OH}$ 
molecules should be linearly related to tip-substrate separation according to the Fick's law of diffusion. The closer to the substrate of the tip electrode is, the higher concentration of $\mathrm{FcCH}_{2} \mathrm{OH}$ molecules becomes. Precisely because of the concentration gradients, the positive feedback current of the approach curves have continuously increased. In order to prove the process of diffusion, the steady-state voltammograms under different tip-substrate separation were performed. The steady-state voltammograms of $0.5 \mathrm{mM} \mathrm{FcCH}{ }_{2} \mathrm{OH}$ at different tip-substrate separation from the 3-MPA SAM modified substrate in $0.2 \mathrm{M} \mathrm{KCl}$ are shown in Figure $4(a)$, the oxidization limiting diffusion current $\left(I_{\infty}\right)$ is gradually increased with the lessening of the tip-substrate separation. The limiting diffusion current is almost linearly growing in the range from 60 to 10 times radius of the tip, seen in Figure 4(b). The increment amount of released $\mathrm{FCCH}_{2} \mathrm{OH}$ molecule is growing with the decrease of the tip-substrate separation distance. This indicates the diffusion of $\mathrm{FcCH}_{2} \mathrm{OH}$ is related to the distance and ensures the appearance of the long-range effect. The steady-state voltammograms of $0.5 \mathrm{mM} \mathrm{FcCH}{ }_{2} \mathrm{OH}$ at different distances from the bare gold substrate in $0.2 \mathrm{M} \mathrm{KCl}$ are shown in Figure $4(\mathrm{c}) . l_{\infty}$ is essentially a constant in the range from 60 to 10 times radius of the tip (Fig. 4d). Also, the steady-state voltammograms of $2 \mathrm{mM} \mathrm{K}_{4}(\mathrm{FeCN})_{6}$ at different distances from the 3-MPA SAM modified substrate in $0.2 \mathrm{M} \mathrm{KCl}$ are shown in Figure $4(\mathrm{e}) . I_{\infty}$ remains a certain value until the tip-substrate separation is less than 5 times radius of the tip (Fig. $4 \mathrm{f}$ ). The results shed light on the explanation for the long-range effect (Scheme 2). It was hard to take the $\mathrm{FcCH}_{2} \mathrm{OH}$ molecules off from the 3-MPA SAM as if they were "glued" to 
the surface by hydrogen bonds. However, the $\mathrm{Fc}^{+} \mathrm{CH}_{2} \mathrm{OH}$ ions generated in front of the tip, would diffuse to the substrate surface thus attracting the carboxylate anions by electrostatic interaction. This leads to the ionization of the carboxyl group, so as to the disruption of the hydrogen bonds between $\mathrm{FcCH}_{2} \mathrm{OH}$ molecules and the 3-MPA SAM. As a result, the additional molecules of $\mathrm{FCCH}_{2} \mathrm{OH}$ would return to the tip which finally causes an enhanced feedback.

We next consider the influence of packing density of the SAM on the long-range effect. The 3-MPA SAMs with different packing densities were formed by controlling the assembly time. And the assembly process was quantitatively investigated by electrochemical quartz crystal microbalance (EQCM). Figure 5(a) shows an exponential decrease of frequency in the first 10 minutes and a nearly linearly decrease of frequency after that. This indicates the formation of 3-MPA SAM on the gold surface, which results in a rapid assembly process in the first 10 minutes and a slow assembly process in the following. The assembly reaction has reached the equilibrium point in 60 minutes. At this equilibrium point, the mass gain of 3-MPA SAM is calculated as $\sim 15 \mathrm{ng}$ based on the changes in frequency. The packing density of 3-MPA SAM (assembling for 60 minutes) on the gold surface calculated from the increase in the mass is $\sim 4 \times 10^{14}$ molecules $\mathrm{cm}^{-2}$, almost equal to the packing density of alkanethiols $\mathrm{CH}_{3}\left(\mathrm{CH}_{2}\right)_{5} \mathrm{SH}$ SAM on gold surface $\left(4.6 \times 10^{14}\right.$ molecules $\left.\mathrm{cm}^{-2}\right)$ [34]. The ionization of the weak acid 3-MPA is feeble in the neutral medium, so the density of the undissociated 3-MPA molecules is approximately equal to the packing density of 3-MPA SAM on the gold surface. And the density of the undissociated 3-MPA 
molecules is equal to the density of hydrogen bonds beforehand formed in the 3-MPA SAM. While the density of untied hydrogen bonds observed in the approach curve calculated by electron charge is $3.4 \times 10^{11}$ molecules $\mathrm{cm}^{-2}$ (integral area above I $\infty$ in Fig. 2(b)). It can be seen that the density of hydrogen bonds beforehand formed in the 3-MPA SAM is obviously higher than the density of untied hydrogen bonds in the enhanced positive feedback, which verifies the mechanism we proposed. Further, the approach curves approaching substrate electrodes with different packing densities of 3-MPA SAM are presented in Figure 5(b). The tip-substrate separation, when the current starts to increase, is increasing with self-assembly time of 3-MPA SAM. This demonstrates the positive correlation between the separation distance and the packing density of the 3-MPA SAM. Now it is clear that why the 3-MPA SAM affects the approach curve. The redox species are beforehand bonded on the SAM by hydrogen bonds. However, the hydrogen bonds will be untied by the ionization of the carboxyl group because the $\mathrm{Fc}^{+} \mathrm{CH}_{2} \mathrm{OH}$ ions generated by the tip attract the negative charged carboxylate ions, leading to the escape of $\mathrm{FcCH}_{2} \mathrm{OH}$ from 3-MPA SAM. And the diffusion of escaped $\mathrm{FcCH}_{2} \mathrm{OH}$ is found to follow the Fick's law of diffusion. As a result, the amount of the released molecules are linear increasing when the tip approaches to the 3-MPA SAM modified substrate, which triggers the long-range effect.

In order to achieve the nondestructive test of living cells using SECM, one-line scanning model of SECM test for HeLa cell on 3-MPA SAM modified substrate was carried out as an example. It has been reported that the redox species function on 
the HeLa cell leads to a positive feedback [25]. In short, the redox species $\mathrm{FcCH}_{2} \mathrm{OH}$ facilitate the intracellular generation of glutathione. The additional glutathione reacts with $\mathrm{Fc}^{+} \mathrm{CH}_{2} \mathrm{OH}$ to generate $\mathrm{FcCH}_{2} \mathrm{OH}$ on the cell membrane, leading to a positive feedback. Figure 6 shows the one-line scanning model over the cell in $0.5 \mathrm{mM}$ $\mathrm{FcCH}_{2} \mathrm{OH}$. The tip was set at $\sim 30 \mu \mathrm{m}$ ( 6 times radius of the tip) above the substrate electrode. The current responses $(\Delta / \mathrm{a})$ for single HeLa cell on the 3-MPA SAM modified substrate were $\sim 68.6 \mathrm{pA}$ while the responses $(\Delta / \mathrm{b})$ for the HeLa cell on the bare substrate were $\sim 17.4 \mathrm{pA}$ (inset of Fig. $6 \mathrm{c}$ ). The current signal for single cell test was obviously enhanced, and the background current on the 3-MPA SAM was also higher than that on the bare substrate. Though the increasing of the background current decreases the resolution, the resolution of single cell with the 3-MPA SAM has been increased. This suggests that the increase of resolution is over compensating the decrease of resolution induced by background current. The current enhancement may be attributed to the additional $\mathrm{FcCH}_{2} \mathrm{OH}$, which is induced by the long-range effect. This observation uncovers the previously unknown property of $\mathrm{FCCH}_{2} \mathrm{OH}$ outside of cells, and demonstrates that the test for the cells with 3-MPA SAM modified substrate was more sensitive than that with bare gold substrate. In addition, the current curve (in black) shows two valleys ( $x=26 \mu \mathrm{m}$ and $\mathrm{x}=46 \mu \mathrm{m}$ ) which is consistent with the results reported by Janine Mauzeroll et al [35]. This is a result from the small perturbations of diffusion triggered by the edge of the cell membrane. It can be seen that the long-range effect induced by 3-MPA SAM provides a reliable approach which allows long range nondestructive detection of single cell. 
Without tip disturbances within the surroundings, the natural behavior of the cells could be precisely sensed by SECM, making the SECM technique practical for clinical use in the future. In addition, it has been confirmed that the cell lines derived from cancers have distinctive morphological property [36], indicating that there are potentials for the development of SECM-based sensors for differentiating cancer cells with normal cells.

\section{Conclusions}

To summarize, we used SECM to quantitatively demonstrate the long-range effect induced by untying hydrogen bonds on the 3-MPA SAM surface. It is found that the hydrogen bonds exist between the carbonyl group of 3-MPA SAM and the hydroxyl group of ferrocenemethanol. The redox species $\mathrm{FcCH}_{2} \mathrm{OH}$ are bonded on the SAM by hydrogen-bonding. However, the hydrogen bonds will be untied by the ionization of the carboxyl group because the $\mathrm{Fc}^{+} \mathrm{CH}_{2} \mathrm{OH}$ ions generated by the tip attract the negative charged carboxylate ions, leading to desorption of $\mathrm{FcCH}_{2} \mathrm{OH}$. On the basis of the discoveries, an approach of SAM modification on the substrate electrode of SECM is proposed, which is applied to the nondestructive test of single living cell.

\section{Acknowledgment}

We gratefully acknowledge the financial support from the National Science Foundation of China $(21173023,21503013,11079010)$, Fundamental Research Funds for the Central Universities (S13JB00200) and National 111 Project of China (B07012). 


\section{References}

[1] A.J. Bard, G. Denuault, C. Lee, D. Mandler, D.O. Wipf, Scanning electrochemical microscopy-a new technique for the characterization and modification of surfaces, Acc. Chem. Res., 23 (1990) 357-363.

[2] Y. Wang, K. Kececi, J. Velmurugan, M.V. Mirkin, Electron transfer/ion transfer mode of scanning electrochemical microscopy (SECM): a new tool for imaging and kinetic studies, Chemical Science, 4 (2013) 3606-3616.

[3] M.E. Abdelhamid, S. Piantavigna, A.M. Bond, B. Graham, L. Spiccia, L.L. Martin, A.P. O'Mullane, An SECM study on the influence of cationic, membrane-active peptides on a gold-supported self-assembled monolayer, Electrochem. Commun., 51 (2015) 11-14.

[4] Y. Takahashi, A.I. Shevchuk, P. Novak, Y. Murakami, H. Shiku, Y.E. Korchev, T. Matsue, Simultaneous noncontact topography and electrochemical imaging by SECM/SICM featuring ion current feedback regulation, J. Am. Chem. Soc., 132 (2010) 10118-10126.

[5] H.S. Ahn, A.J. Bard, Surface Interrogation of CoPi Water Oxidation Catalyst by Scanning Electrochemical Microscopy, J. Am. Chem. Soc., 137 (2015) 612-615.

[6] B.H. Simpson, J. Rodríguez-López, Redox Titrations via Surface Interrogation Scanning Electrochemical Microscopy at an Extended Semiconducting Surface for the Quantification of Photogenerated Adsorbed Intermediates, Electrochim. Acta, 179 (2015) 74-83.

[7] J.n. Rodríguez-López, M.A. Alpuche-Avilés, A.J. Bard, Interrogation of Surfaces for the Quantification of Adsorbed Species on Electrodes: Oxygen on Gold and Platinum in Neutral Media†, J. Am. Chem. Soc., 130 (2008) 16985-16995.

[8] J.C. Love, L.A. Estroff, J.K. Kriebel, R.G. Nuzzo, G.M. Whitesides, Self-assembled monolayers of thiolates on metals as a form of nanotechnology, Chem. Rev., 105 (2005) 1103-1169.

[9] A. Ulman, Formation and structure of self-assembled monolayers, Chem. Rev., 96 (1996) 1533-1554.

[10] T. Wilhelm, G. Wittstock, Localised electrochemical desorption of gold alkanethiolate monolayers by means of scanning electrochemical microscopy (SECM), Microchimica Acta, 133 (2000) 1-9.

[11] E. Malel, J. Colleran, D. Mandler, Studying the localized deposition of Ag nanoparticles on self-assembled monolayers by scanning electrochemical microscopy (SECM), Electrochim. Acta, 56 (2011) 6954-6961.

[12] S. Ido, H. Kimiya, K. Kobayashi, H. Kominami, K. Matsushige, H. Yamada, Immunoactive two-dimensional self-assembly of monoclonal antibodies in aqueous solution revealed by atomic force microscopy, Nat Mater, 13 (2014) 265-271.

[13] X.Y. Cheng, S.B. Lowe, P.J. Reece, J.J. Gooding, Colloidal silicon quantum dots: from preparation to the modification of self-assembled monolayers (SAMs) for bio-applications, Chem. Soc. Rev., 43 (2014) 2680-2700.

[14] X. Chen, C.Y. Hong, Y.H. Lin, J.H. Chen, G.N. Chen, H.H. Yang, Enzyme-Free and Label-Free Ultrasensitive Electrochemical Detection of Human Immunodeficiency Virus DNA in Biological Samples Based on Long-Range Self-Assembled DNA Nanostructures, Anal. Chem., 84 (2012) 8277-8283.

[15] M. Burchardt, G. Wittstock, Kinetic studies of glucose oxidase in polyelectrolyte multilayer films by means of scanning electrochemical microscopy (SECM), Bioelectrochemistry, 72 (2008) 66-76.

[16] R.K. Shervedani, M.S. Foroushani, Comparative Electrochemical Behavior of Proteins; Cytochrome c, Agaricus Bisporus Laccase, and Glucose Oxidase, Immobilized onto Gold-Thiol Self-Assembled Monolayer via Electrostatic, Covalent, and Covalent Coordinate Bond Methods, Electrochim. Acta, 187 
(2016) 646-654.

[17] R.G. Fedorov, D. Mandler, The Effect of Self-Assembled Monolayers on the Locally Electrodeposited Silver Thin Layers, The Journal of Physical Chemistry C, (2016). DOI: 10.1021/acs.jpcc.5b06680

[18] C.D. Hodneland, Y.-S. Lee, D.-H. Min, M. Mrksich, Selective immobilization of proteins to self-assembled monolayers presenting active site-directed capture ligands, Proceedings of the National Academy of Sciences, 99 (2002) 5048-5052.

[19] W.M. Shih, Exploiting weak interactions in DNA self-assembly, Science, 347 (2015) 1417-1418.

[20] W.L. Davis, R.F. Shago, E.H. Langner, J.C. Swarts, Synthesis and electrochemical properties of a series of ferrocene-containing alcohols, Polyhedron, 24 (2005) 1611-1616.

[21] N.K. Chaki, K. Vijayamohanan, Self-assembled monolayers as a tunable platform for biosensor applications, Biosens. Bioelectron., 17 (2002) 1-12.

[22] J.J. Gooding, S. Ciampi, The molecular level modification of surfaces: from self-assembled monolayers to complex molecular assemblies, Chem. Soc. Rev., 40 (2011) 2704-2718.

[23] T. Sawaguchi, Y. Sato, F. Mizutani, In situ STM imaging of individual molecules in two-component self-assembled monolayers of 3-mercaptopropionic acid and 1-decanethiol on Au (111), J. Electroanal. Chem., 496 (2001) 50-60.

[24] G. Sauerbrey, Verwendung von Schwingquarzen zur Wägung dünner Schichten und zur Mikrowägung, Zeitschrift für physik, 155 (1959) 206-222.

[25] S. Kuss, R. Cornut, I. Beaulieu, M.A. Mezour, B. Annabi, J. Mauzeroll, Assessing multidrug resistance protein 1-mediated function in cancer cell multidrug resistance by scanning electrochemical microscopy and flow cytometry, Bioelectrochemistry, 82 (2011) 29-37.

[26] M. Petri, D.M. Kolb, U. Memmert, H. Meyer, Adsorption of mercaptopropionic acid onto Au (1 11 1): Part I. Adlayer formation, structure and electrochemistry, Electrochim. Acta, 49 (2003) 175-182.

[27] S.-f. Liu, X.-h. Li, Y.-c. Li, Y.-f. Li, J.-r. Li, L. Jiang, The influence of gold nanoparticle modified electrode on the structure of mercaptopropionic acid self-assembly monolayer, Electrochim. Acta, 51 (2005) 427-431.

[28] Q. Zheng, H. Wu, Z. Shen, W. Gao, Y. Yu, Y. Ma, W. Guang, Q. Guo, R. Yan, J. Wang, K. Ding, An electrochemical DNA sensor based on polyaniline/graphene: high sensitivity to DNA sequences in a wide range, Analyst, 140 (2015) 6660-6670.

[29] E. Sabatani, I. Rubinstein, Organized self-assembling monolayers on electrodes. 2. Monolayer-based ultramicroelectrodes for the study of very rapid electrode kinetics, J. Phys. Chem., 91 (1987) 6663-6669.

[30] F. Malem, D. Mandler, Self-assembled monolayers in electroanalytical chemistry: application of. omega.-mercapto carboxylic acid monolayers for the electrochemical detection of dopamine in the presence of a high concentration of ascorbic acid, Anal. Chem., 65 (1993) 37-41.

[31] B. Liu, A.J. Bard, M.V. Mirkin, S.E. Creager, Electron transfer at self-assembled monolayers measured by scanning electrochemical microscopy, J. Am. Chem. Soc., 126 (2004) 1485-1492.

[32] R. Cornut, C. Lefrou, A unified new analytical approximation for negative feedback currents with a microdisk SECM tip, J. Electroanal. Chem., 608 (2007) 59-66.

[33] J. Kim, J.L. Connell, M. Whiteley, A.J. Bard, Development of a Versatile in Vitro Platform for Studying Biological Systems Using Micro-3D Printing and Scanning Electrochemical Microscopy, Anal. Chem., 86 (2014) 12327-12333.

[34] D.M. Collard, M.A. Fox, Use of Electroactive Thiols to Study the Formation and Exchange of 
Alkanethiol Monolayers on Gold, Langmuir, 7 (1991) 1192-1197.

[35] S. Kuss, D. Trinh, L. Danis, J. Mauzeroll, High-Speed Scanning Electrochemical Microscopy Method for Substrate Kinetic Determination: Method and Theory, Anal. Chem., 87 (2015) 8096-8101.

[36] A.F. Gazdar, D.N. Carney, M.M. Nau, J.D. Minna, Characterization of Variant Subclasses of Cell-Lines Derived from Small Cell Lung-Cancer Having Distinctive Biochemical, Morphological, and Growth-Properties, Cancer Research, 45 (1985) 2924-2930. 
Scheme 1



Figure 1
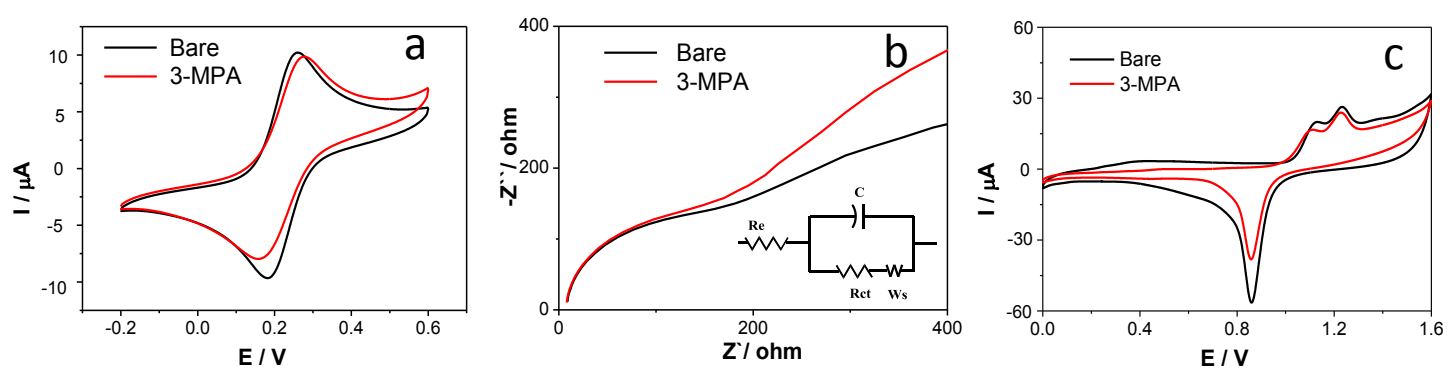
Figure 2
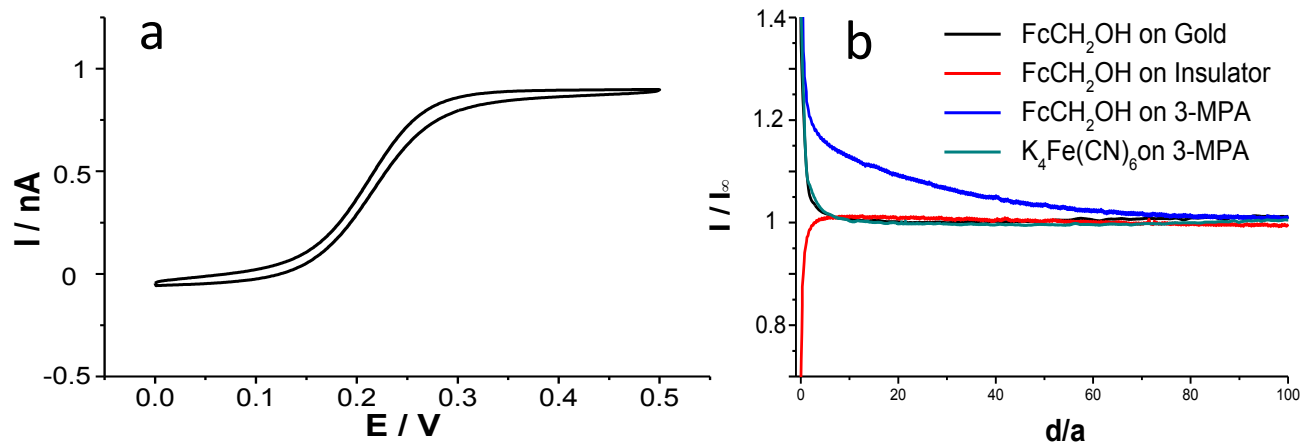

Figure 3
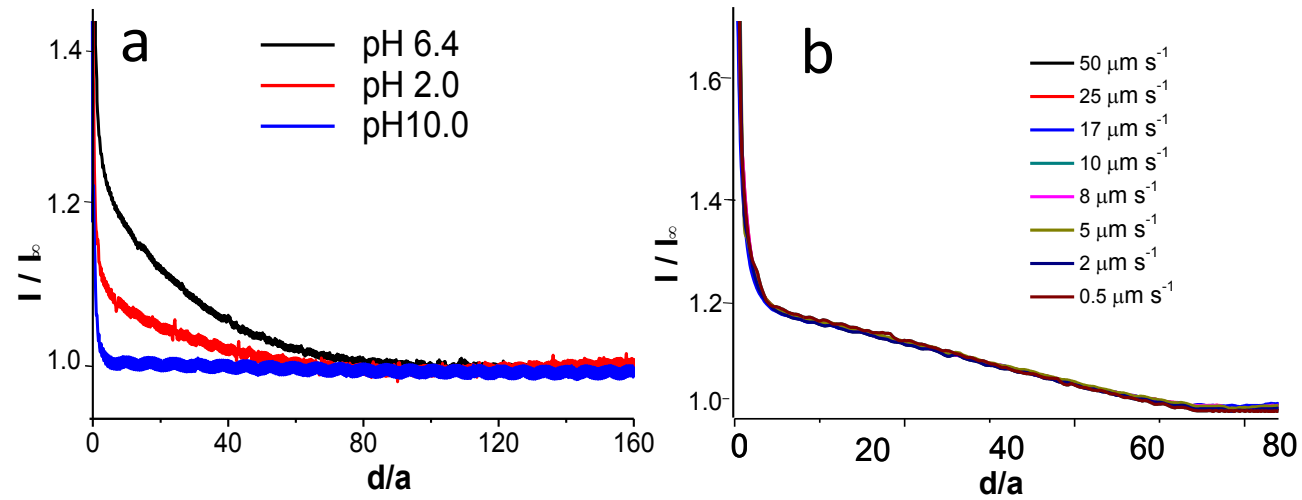
Figure 4


Scheme 2
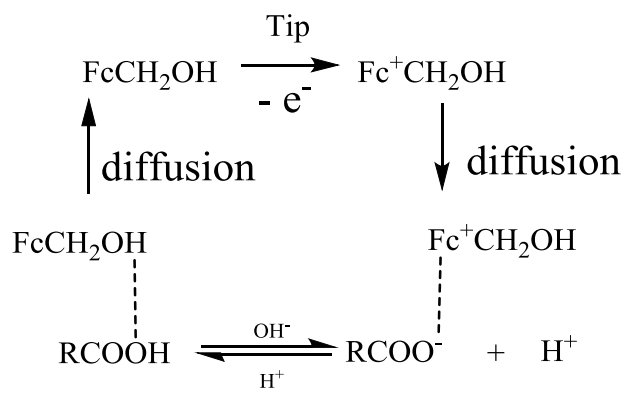

hydrogen-bonding electrostatic attraction 
Figure 5
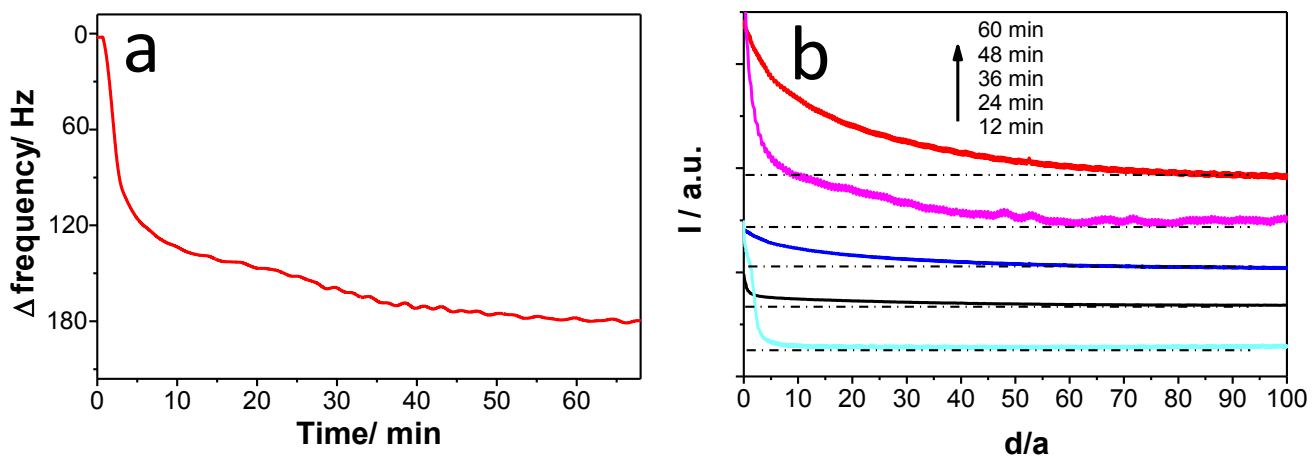

Figure 6

Graphical Abstract (for review)



